\title{
New methods to assess fecal contamination in beach water quality
}

\begin{abstract}
The emerging paradigm in assessment of recreational water quality needs a basic information on microbial contamination. Recreational water is susceptible to fecal contamination which may increase in health risk associated with swimming in polluted water. Recreational water quality is monitored for fecal indicator bacteria to help prevent swimming-associated illnesses. This study aims to determine the concentrations of total coliforms and Escherichia coli (E. coli ) in recreational water, Teluk Kemang beach, Port Dickson (Malaysia). This study was also aimed to determine relationship between total coliforms, E. coli and physicochemical parameters of marine recreational water. Exposure behaviors and perceived health symptoms among beach visitors were also assessed in this study. A total of eight water sampling points were selected randomly taken at $100 \mathrm{~m}$ from the wash zone and $20 \mathrm{~cm}$ below the water surface along Teluk Kemang beach. Total coliforms and E. coli concentrations were analyzed using membrane filtration technique. Total coliforms concentrations were found between 20 and $1,940 \mathrm{cfu} / 100 \mathrm{ml}$. E. coli concentrations were between 0 and 90 cfu/100 ml. Total coliforms and E. coli were the highest at sampling point A and exceeded USEPA (Report of Task Force on guide standard and protocol for testing microbiological water purifiers. USEPA, Washington, DC, pp 1-29, 1986) guideline as it located near sewage out-fall. Significant correlations were found between total coliforms and E. coli with $\mathrm{pH}$, temperature and oxidation reduction potential. Microbiological water quality in Teluk Kemang public beach was generally safe for recreational activities among the public except sampling location near with sewage outfall. Output of this study will act as a crucial model for protection of marine ecosystem health. Moreover, output of this study can be used as a monitoring design tool for other public beaches in Malaysia, as such studies are lacking and have not been reported so far.
\end{abstract}

Keyword: Recreational water; Water quality; Escherichia coli (E. coli ) 